\title{
La heurística y la toma de decisiones en empresas de servicios ${ }^{a}$
}

\section{The heuristic and the decision making in services companies Heurística e a toma de decisão em empresas de serviços}

\author{
Magíster Germán Rubio Guerrero* \\ Magíster Fernando Adolfo Fierro Celis**
}

\section{RESUMEN}

El propósito de la investigación fue estudiar los prejuicios y errores en la toma de decisiones y su relación con los modelos racionales en 16 empresas de servicios seleccionadas a juicio de los investigadores en los departamentos de Tolima y Huila, Colombia. Se trató de un estudio mixto que a través del análisis multidimensional, permitió establecer que en estas organizaciones prevalecen los enfoques intuitivos sobre los formales en sus procesos decisionales. Igualmente se evidenciaron correlaciones significativas e independencia entre estas variables.

Palabras clave: toma de decisiones, decisiones estratégicas, racionalidad, racionalidad limitada, métodos para la toma de decisiones, incertidumbre.

\begin{abstract}
The purpose of this research was to study the prejudices and mistakes in decision making and your relation with the rational models in 6 enterprises of services selected to judgment of the investigators on the departments of Tolima and Huila, Colombia. It was a combined study which through of multivariate analysis, allowed to establish that in these organizations prevail the intuitive approaches over that the formals in their decision making process. Equally significant correlations between thesevariablesand independence were evident.
\end{abstract}

Keywords: decisions making, strategic decisions, heuristic, rationality, bounded rationality, methods making decisions, uncertainty.

a Artículo de investigación derivado del proyecto "Habilidades gerenciales en las empresas", adscrito al Grupo de Investigación en Desarrollo Económico y Empresarial de la Facultad de Ciencias Económicas y Administrativas de la Universidad del Tolima-GIDEUT, clasificado “D” Colciencias. Proyecto desarrollado en el año 2013. Clasificación JEL: M1, M10.

* Colombiano, Administrador de Empresas, Magíster en Administración, Doctorando en Gestión, profesor de planta, Universidad del Tolima, Ibagué, Colombia. Correspondencia con el autor: grubio@ut.edu.co.

** Colombiano, Administrador de Empresas, Magíster en Administración, Doctorando en Gestión, profesor de planta, Universidad Surcolombiana, Neiva, Colombia. Correspondencia con el autor: fernando.fierro@usco.edu.co. 


\section{RESUMO}

O objetivo da pesquisa foi estudar os preconceitos e erros na toma de decisões e sua relação com os modelos racionais em 16 empresas de serviços, selecionadas segundo o parecer dos pesquisadores dos departamentos de Tolima e Huila, na Colômbia. Este foi um estudo misto que a través da análise multidimensional, permitiu estabelecer que nestas organizaçóes prevalecem os enfoques intuitivos por sobre os formais em seus processos de toma de decisão. Da mesma forma, se evidenciaram correlaçóes significativas e independência entre estas variáveis.

Palavras-chave: toma de decisóes, decisões estratégicas, racionalidade, racionalidade limitada, métodos de toma de decisão, incerteza.

\section{Introducción}

Este artículo presenta un análisis del proceso de toma de decisiones en un grupo de organizaciones públicas y privadas del sector de los servicios seleccionadas por conveniencia de los investigadores en los Departamentos de Tolima y Huila, Colombia, e hizo parte del trabajo de investigación adelantado por el Grupo de Investigación en Desarrollo Económico y Empresarial (GIDEUT) de la Universidad del Tolima. Se analizaron de manera especial los 12 prejuicios y errores comunes en la toma de decisiones presentados por Robbins y Coulter (2010:133), dentro de la perspectiva de Minztberg (1997:43) cuando circunscribe al administrador como el encargado de tomar decisiones en sus facetas de emprendedor, manejador de conflictos, asignador de recursos y negociador, y de March y Simon (1981:151), que exponen que quien toma una decisión tiene una función de utilidad y una preferencia de ordenación, que jerarquiza todos los conjuntos de consecuencias, desde los más preferidos a los menos preferidos.

El estudio se consideró mixto en tanto que incorpora realidades objetivas, subjetivas e intersubjetivas e incorpora de hecho los enfoques cuantitativo y cualitativo (Hernández et al. 2010:25 y Deslauriers, 2004:20). La información se recolectó mediante un cuestionario estructurado y para el tratamiento de los datos se emplearon las técnicas de análisis descriptivo, pruebas de independencia y escalamiento multidimensional del análisis multivariado con el programa SPSS.

\section{Marco teórico}

Una decisión no es un hecho simple, unitario, sino el producto de un proceso social complejo generalmente extendido sobre un considerable período de tiempo. En este sentido la toma de decisiones incluye procesos de atención, dirección y de inteligencia que determinan las oportunidades de la decisión. Procesos para 
descubrir y diseñar posibles cursos de acción y procesos para evaluar alternativas y seleccionar entre ellas.

Dentro de este contexto (Simon, 1965:31,32), a propósito de los métodos cuantitativos para la toma de decisiones, argumentó que estas herramientas, en especial las de investigación de operaciones, tal vez han tenido más aplicación en los negocios que en las organizaciones gubernamentales. La investigación de operaciones y la ciencia administrativa han contribuido de muchas maneras a la teoría de la toma de decisiones. La meta ha sido diseñar herramientas que ayuden a la gerencia a tomar mejores decisiones, un ejemplo de ello es el procedimiento de programación llamado PERT o programación de la ruta crítica. Igualmente las decisiones organizacionales pueden derivarse de experimentos debidamente planeados con individuos o con pequeños grupos de personas como el caso de Hawthorne, la heurística y la técnica de la persuasión y evocación, entre otras.

Desde el punto de vista tradicional el proceso de toma de decisiones se ha realizado mediante el sentido común usando un lenguaje cotidiano. La noción de que una decisión puede ser vista como una conclusión extraída de premisas, hace necesario encontrar las fuentes de las premisas y los canales de comunicación que ellos siguieron a través de la organización hasta el punto donde ellas se conviertan en la materia prima de la decisión. Los estudios que han adoptado este enfoque general para la descripción de las decisiones dentro del método del estudio de casos, se hicieron cada vez más frecuentes durante el período objeto de análisis (Simon, 1965). Un ejemplo es el excelente estudio del guardabosque de Herbert Kaufman, dirigido a analizar "la forma en que sus decisiones y comportamientos son influenciados dentro y por el servicio". Otro es el estudio dirigido por el Grupo Carnegie Tech sobre la influencia de la información contable sobre las decisiones de operación en las grandes compañías.

Con base en el concepto de premisa (Simon, 1965; Machado, Van \& Britto, 2013:58), experimentaron con estímulos estandarizados para modelar problemas que demandaran una decisión a manera de conflictos (conflictos de tipo aproximación-evitación, aproximación-aproximación y evitación-aproximación). Un resultado que surgió del experimento es la percepción de las personas adheridas significativamente a la tarea y sin renuencia para tratar de averiguar lo imponderable. ¿Por qué nadie se queja o actúa como si la tarea fuera imponderable? Esta es una muy interesante y propia tendencia psicológica, con la cual se especula para concluir que más personas tienden a tener una limitación general en ver cosas como imponderables o aleatorias, coincidiendo con Robbins y Coulter (2010:134) cuando a instancias de los prejuicios y errores en la toma de decisiones exponen el criterio del "error de causalidad", significando que los 
tomadores de decisiones intentan darle sentido a eventos casuales y lo hacen debido a que la mayoría tiene problemas para lidiar con el azar.

La intuitividad de la decisión se relaciona con el nivel de stress que eso evoca, siempre que el problema como un todo no sea tratado analíticamente; esto es, una toma de decisiones intuitivas involucra un estilo de decisión por el cual el agente usa su atracción espontánea en las diferentes opciones, mientras no las analiza hipotética y deductivamente como un criterio principal de decisión. Esta situación se ilustra un poco más con el experimento de Bechara et al. (2005), citado por Machado et al. (2013:59, 60), quienes demostraron que después de la selección de aproximadamente la mitad de las cartas (durante un juego involucrando 4 barajas y un total de 100 cartas), la gente empieza a mostrar una notable preferencia hacia las pilas con el más alto pago total (en otras palabras ellos empiezan a decidir ventajosamente) y que esta tendencia tiende a estar presente independientemente de su incapacidad de ranquear las pilas en términos de su utilidad global.

Según Regent (2012:82), en el mundo de la empresa es posible distinguir a los directivos que se apoyan en su intuición de aquellos otros que se basan en metodologías formales. Los primeros suelen hacerse notar debido a que toman decisiones sin entretenerse demasiado en análisis de ningún tipo. Son los que la "tienen clara", los "que la ven". Los segundos se destacan por tomarse su tiempo para recabar información y pedir análisis que sustenten sus procesos decisorios. No acostumbran decidir antes de ejecutar una serie de pasos que les asegura que no han dejado nada por el camino. Según Regent (2012), los intuitivos suelen llevar ventaja cuando se encuentran en entornos estables. Los metódicos logran un mejor desempeńo cuando es necesario decidir en entornos poco conocidos o sujetos a cambios en una o más de las variables relevantes. Concluye Regent (2012) que cuando hay que usar el criterio, lo propio de la función directiva es emplear primero el método y luego eso que el común de la gente llama intuición. Siempre que enfrente un problema, aplique todos los análisis técnicos a su alcance para delimitar el universo posible; una vez que estos no den más de sí, no siga buscando análisis alternativos, asuma que ha llegado el momento de decidir, el tiempo político.

Según Círigo (2010:72), la intuición comprende varios sinónimos: visión, percepción, vislumbre, conocimiento, clarividencia, discernimiento, idea, perspicacia, sagacidad, sospecha, adivinación, presentimiento, sexto sentido, y en términos coloquiales, "latido", "corazonada", "punzada”, "lo siento”, "me late”, "vibra”, etc. Gerd Gigerenzer, profesor y director del Centro de Comportamiento Adaptativo y Cognición del Instituto Max Planck para el Desarrollo Humano de Berlín, citado por Círigo (2012:72), sostiene que a menudo la inteligencia funciona sin un pensamiento consciente y todavía más, la corteza cerebral, donde supuestamente reside la conciencia, está plagada de procesos 
inconscientes, por lo que sería temerario afirmar que la inteligencia es siempre consciente y reflexiva.

En esta misma dirección, Dane \& Pratt (2007:48) afirman que más allá de la toma de decisiones, la intuición puede tener otros beneficios positivos también. Esto se ilustra con algunos trabajos que sugieren una asociación entre intuición y creatividad. Por ejemplo afirman, citando a Langer (1989:177), que la creatividad se origina a través de "una experiencia intuitiva del mundo" mientras que el pensamiento racional sirve solo para confirmar "mentalidades antiguas" y "categorías rígidas" coincidiendo con Poincaré (1969:210), también citado por Dane \& Pratt (2007:48), cuando expone que la "lógica es el instrumento de demostración" y la intuición el "instrumento de la invención".

Según Dane \& Pratt (2007:41, 49), la intuición es buena para decisiones importantes que involucren estrategias, inversiones y gestión del talento humano (Miller \& Ireland, 2005: 19, 28), cuando existe la necesidad por decisiones rápidas y condiciones cambiantes del entorno (González, 2012:17), pero no en otras como es el caso de la solución de problemas matemáticos y tareas muy estructuradas que pueden conducir a soluciones muy imprecisas. La intuición es, por consiguiente, esencial para la vida y para la gerencia, y aunque no existe consenso puede relacionarse con percepción inconsciente, instinto, reconocimiento de patrones, procesamiento automático, activación automática, corte fino (concluir con poca información), conocimiento tácito, cognición encarnada, corazonadas o emoción como fuente de información (González, 2012:18; Círigo, 2010:72).

En otros términos, pero coincidiendo con la mayoría de las afirmaciones anteriores, Ramsés y Obuchi (2010:23) plantean que la toma de decisiones se enfoca desde dos perspectivas, una prescriptiva o normativa asociada con la racionalidad, la lógica y los modelos formales, y la descriptiva vinculada con procesos sicológicos, conductas e intuiciones. En esta perspectiva coinciden con Miller \& Ireland (2005:19, 28), en el sentido que también manifiestan que ambos enfoques se solapan, ya que los modelos formales del enfoque normativo necesitan aproximaciones intuitivas para ajustar ciertos parámetros y supuestos; y las decisiones de tipo descriptivo recurren a modelos formales y semiformales para entender y facilitar la toma de decisiones desde el punto de vista conductual (Simon, 1987). Al decidir se ponen en juego todas las facultades humanas: desde la racionalidad hasta la intuición y las emociones; desde la naturaleza gregaria del ser humano, que le permite utilizar su inteligencia colectiva y colaborativa, hasta el aislamiento del pensador solitario; desde el pensamiento crítico hasta la alegre improvisación de las tormentas de ideas; desde el anhelo de percepción hasta la propensión a cometer errores que permiten descubrir soluciones novedosas e insospechadas. Entre los sesgos clásicos que afectan a los gerentes en el proceso de toma de decisiones están 
el sesgo de confirmación y las ilusiones positivas. El primero consiste en la tendencia de buscar información que confirme las propias ideas, hipótesis, creencias o preconcepciones, y obviar la información que las desmienta. Las ilusiones positivas son sesgos que se asocian con visiones irreales y favorables de uno mismo (exceso de confianza y optimismo excesivo).

Estos sesgos van en la misma dirección de Robbins y Coulter (2010:133), cuando exponen los prejuicios y errores en la toma de decisiones, particularmente el exceso de confianza y la confirmación e igualmente la heurística de la representatividad al creer que situaciones aisladas pueden ser generalizadas (González, 2012:20). Las intuiciones desde un punto de vista práctico se constituyen en el punto de partida de la formulación de hipótesis, las cuales a través de la información y el control respectivo son contrastadas y las que sobreviven engrosan el conocimiento científico (González, 2012:21).

Desde otra perspectiva, Langley, Mintzberg, Pitcher, Posada \& Macary (1995:260, $276,277)$ analizan la toma de decisiones desde tres aspectos: la apertura a la ambigüedad o la relación entre el compromiso y la acción; el tomador de decisiones está abierto a la historia y la experiencia para afectar, inspirar y especialmente para criticar el rol de conocimiento, para trascender los límites de la racionalidad cerebral, y por último el proceso de toma de decisiones es abierto para organizar un vínculo dinámico de manera que trazos aislados de decisiones singulares vienen a ser vistas como entretejidos de asuntos de redes. En este sentido sugieren las siguientes premisas: la primera de ellas "trace asuntos hacia adelante y no decisiones hacia atrás"; en segundo lugar "intente nuevas perspectivas, acercar o alejar la imagen” y en tercera instancia "seguir los procesos en tiempo real, así como retrospectivamente", que sugiere una relación con los dos aspectos anteriores. Esto asegura que las percepciones no son sesgadas por un conocimiento de un resultado final o efecto ancla (Robbins y Coulter, 2010:133).

Una cuarta premisa es el "enfoque sobre la gente y su personalidad, no solo los eventos"; en este contexto la literatura ha adoptado frecuentemente una muy estrecha visión del tomador de decisiones; no solo se han descuidado ciertas facultades importantes tales como la perspectiva y la inspiración, sino también ha tendido a ignorar diferencias individuales. La última premisa es "reanalizar previamente los procesos de decisión estudiados no solo otros nuevos", que expone una manera para probar nuevas ideas que podría ser volver a decisiones previamente analizadas para determinar si las nuevas propuestas aquí pueden ayudar en alguna cosa para nuestro entendimiento. Este aspecto, en términos de Robbins y Coulter (2010:134), se denomina errores de representación y se da cuando los tomadores de decisiones evalúan la posibilidad de un evento guiándose por el parecido con otro evento o series de eventos. 
Según Murphy (1992:293, 302), la postura de Simon sobre su concepto de la racionalidad limitada es aceptada, pues no puede ser diferenciada de la interpretación, puesto que esta es finita y así la razón no es universal. En este sentido mejorar metodologías, expandir las bases de datos y la asistencia de expertos técnicos no necesariamente mejorará la toma de decisiones. Dentro de este mismo contexto, Forest y Mehier (2001:591) analizaron las propuestas de Commons y Simon, concluyendo que el modelo de racionalidad de Simon es muy cercano a la visión de Commons, en especial al aspecto relacionado con la atención como parte de la racionalidad. Sin embargo, el trabajo de Simon va mucho más allá de Commons, argumentando que el objeto de Commons y Simon no es demostrar que los individuos son seres irracionales en su toma de decisiones, pues Commons vio a los individuos más direccionados por los hábitos, instintos y pasiones que por la razón, y en este sentido usó el término conductivismo, lo que corrobora lo expresado en los apartados anteriores por otros autores acerca de la importancia de la intuitividad en los procesos de toma de decisiones. Simon, desde 1934, observó que las decisiones no son hechas de manera standard como sugiere la teoría; igualmente refutó la hipótesis de información pura y perfecta y de racionalidad perfecta. Forest \& Mehier (2001:597) observan así mismo con Commons y Simon que el medio ambiente de los individuos determina su comportamiento en conjunto.

A propósito de los efectos directos y las interacciones entre la magnitud de las consecuencias y varios tipos de proximidad: social, sicológica y física sobre los procesos de toma de decisiones éticas y la influencia de la empatía sobre los mismos, Mend \& May (2009:201) encontraron en una muestra de gerentes de talento humano, que estos directivos tendieron a reportar altos niveles de empatía cognitiva pero bajos niveles de empatía afectiva. Así mismo argumentan que la toma de decisiones es un proceso complejo y desde la perspectiva económica las decisiones óptimas son todas aquellas que tienen fundamento racional, es decir, se asume que los tomadores de decisiones disponen de toda la información y conocen todas las posibles alternativas y consecuencias y que no están influenciados por otros en su meta de maximizar los resultados (Zey, 1992). De hecho esta no es la situación frecuente y los investigadores han estudiado cómo las restricciones situacionales ejercen influencia sobre el proceso de toma de decisiones, tales como el tiempo y la incertidumbre.

De otra parte, Tsang (2004:92), en un análisis que hizo sobre la relación entre la superstición y la toma de decisiones en las comunidades de negocios chinos, expone que aunque la superstición constituye una parte crucial en la vida de negocios de las sociedades chinas, la literatura administrativa ha estado prácticamente silenciosa con respecto a este fenómeno. De una parte, la irracionalidad normalmente asociada con la superstición y la supuesta racionalidad de la toma de decisiones sugiere una aparente contradicción entre las dos. De la misma manera la superstición y la toma de decisiones 
pueden ser complementarias, en el sentido que la primera puede ser usada como un medio para hacer frente a la incertidumbre asociada con esta última. La complementariedad sugiere que la superstición cumple dos funciones principales: proveer una fuente adicional de información y reducir la incertidumbre inducida por la ansiedad.

Este hecho desde luego involucra ciertos costos, ya que la superstición, por definición, no se soporta en evidencia científica, una práctica de este tipo puede ser completamente absurda. El riesgo de basar una decisión en tal práctica es obvio. Por ejemplo se encontró en el estudio de Tsang (2004:103), que muchos gerentes japoneses buscan asesoría de "Ebiso", el dios sintoísta de los negocios y unos de los siete sintoístas de la buena suerte. Cerca de 96 millones de japoneses reclaman ser sintoístas. Los gerentes indios son supersticiosos también como se evidencia por la popularidad de Vaastu Shastra, una práctica supersticiosa que intenta crear armonía entre los cinco elementos ambientales críticos: tierra, cielo, fuego, agua y aire. Es importante destacar que los indios, los chinos y los japoneses juntos constituyen la mayoría de la población de Asia y cerca del $40 \%$ de la población mundial.

Según Rivera, Braunstein \& Hall (2004:116), en un estudio acerca del procesamiento de información gerencial, abordaron aspectos concernientes con la idoneidad y oportunidad de las teorías del comportamiento en la toma de decisiones, mediante el direccionamiento conceptual y metodológico basado en el estudio de procesos cognitivos individuales. El estudio de cognición o de procesos de pensamiento ha sido categorizado como normativo y descriptivo (Círigo, 2010; Regent, 2012; Dane \& Pratt, 2007; Ramsés y Obuchi, 2010; Simon, 1987; Langley, Mintzberg, Pitcher, Posada \& Macary, 1995). Es normativo cuando prescribe cursos de acción que están más cercanos a valores y creencias. La investigación predominante ha sido el uso del teorema de Bayes, en el cual el objetivo ha sido conseguir que las personas expresen opiniones en términos de probabilidades subjetivas y revisarlas sistemáticamente a la luz de nueva información usando el teorema.

Desde el punto de vista descriptivo analiza cómo los procesos de información individuales permiten llegar a decisiones. Entre ellos se destacan los enfoques correlacional y regresional, que intentan representar estrategias de procesamiento de información como modelos matemáticos de decisiones actuales, con el fin de predecir resultados en varias tareas de juicio, como por ejemplo modelos lineales aditivos que han sido usados para predecir resultados en una amplia variedad de áreas, incluyendo evaluación financiera, selección de portafolios, diagnóstico médico y comportamiento de elección del consumidor. A pesar de las contribuciones hechas por estos modelos, su utilidad en la explicación de los procesos de juicio ha sido limitada por problemas de interpretación y una inadecuada descripción de los aspectos subyacentes. 
Con relación a las condiciones que inciden en la toma de decisiones, Franklin (2011:103) cita a la certidumbre, el riesgo y la incertidumbre (Castillo, 2006:20, 21). El primer caso se da cuando se conocen los resultados con certeza en el momento en que se toma la decisión, es decir, se trata de fenómenos determinísticos propios del mundo racional y normativo. A medida que la información disminuye y se torna ambigua, la condición de riesgo entra en el proceso, las consecuencias de esas decisiones no se conocen con certeza en el momento de tomarlas, y en la condición de incertidumbre, las personas que toman una decisión cuentan con poca información o ninguna y sus resultados no se conocen con certeza en el momento en que se toma la decisión. En estos dos últimos estados de cosas se encuentran inmersos la intuición y los modelos descriptivos de toma de decisiones.

\section{Metodología}

\subsection{Alcance de la investigación y recolección de datos}

El artículo es producto de una investigación acerca de las características del proceso de toma de decisiones en empresas de servicios seleccionadas por conveniencia y juicio de los investigadores en los Departamentos del Tolima y Huila en el año 2013, realizada por parte del grupo de investigación GIDEUT de la Universidad del Tolima. A estas empresas se les aplicó un cuestionario estructurado de objetivo claro, que contenía las variables relacionadas con los prejuicios y errores en la toma de decisiones en la perspectiva de Robbins y Coulter (2010:133) (ver tabla 1). Se trató de un estudio mixto en términos de Deslauriers (2004:19), en tanto que midió un fenómeno social, que ofrece una expresión cifrada a los datos y los analiza con la ayuda de métodos estadísticos, coincidiendo con Hernández (2010:77), cuando expone que los estudios con este enfoque pueden ser exploratorios, descriptivos, correlacionales y explicativos.

El siguiente es el sistema de hipótesis que fueron sometidas a prueba en la investigación:

$\mathbf{H}_{01}$ : los prejuicios y errores en la toma de decisiones están correlacionados positivamente en las organizaciones objeto de estudio.

$\mathbf{H}_{1}$ : los prejuicios y errores en la toma de decisiones no están correlacionados positivamente en las empresas estudiadas.

$\mathbf{H}_{02}$ : cada uno de los errores y prejuicios tienen el mismo nivel de correlación en el proceso de toma de decisiones en estas organizaciones. 
$\mathbf{H}_{2}$ : cada uno de los errores y prejuicios tienen diferente nivel de correlación en el proceso de toma de decisiones en estas organizaciones.

$\mathrm{H}_{03}$ : los procesos intuitivos son independientes en la toma de decisiones de las empresas analizadas.

$\mathbf{H}_{3}$ : los procesos intuitivos no son independientes en la toma de decisiones de las empresas objeto de estudio.

Tabla 1. Prejuicios y errores en la toma de decisiones.

\begin{tabular}{|c|c|c|}
\hline No. Variable & Descripción & Código \\
\hline 1 & Exceso de confianza & EXCON \\
\hline 2 & Satisfacción inmediata & SATIN \\
\hline 3 & Efecto ancla & EFEAN \\
\hline 4 & Percepción selectiva & PERSEL \\
\hline 5 & Confirmación & CONFIR \\
\hline 6 & Contexto & CONTEX \\
\hline 7 & Disponibilidad & DISPON \\
\hline 8 & Representación & REPRES \\
\hline 9 & Casualidad & CASUAL \\
\hline 10 & Costos irrecuperables & COSIRR \\
\hline 11 & Egoísmo & EGOIS \\
\hline 12 & Retrospectiva & RETROS \\
\hline
\end{tabular}

Fuente: elaboración propia a partir de Robbins y Coulter (2010).

Con respecto a la contrastación empírica de consistencia, validez y confiabilidad, se obtuvieron respuestas de 16 empresas sobre un total de 28 contactadas, lo cual arrojó un porcentaje de respuesta del $57 \%$. El instrumento utilizado en la investigación ha sido empleado en estudios similares sobre caracterización de procesos organizacionales y de responsabilidad social empresarial dentro del grupo de investigación en desarrollo económico y empresarial de la Universidad del Tolima, "GIDEUT”. La validez de contenido se basó en la revisión de antecedentes y literatura relacionada con el tema, la experiencia y juicio de los docentes y una prueba piloto a 4 de las empresas seleccionadas para comprobar que las preguntas del cuestionario fueran claras para los encuestados. La validez convergente se realizó a través de la matriz de correlaciones entre las diversas dimensiones del cuestionario y se obtuvo que las correlaciones son significativas al 1\% y al 5\% y su determinante es de 3,544E-005, que es muy bajo e indica que las variables están bastante correlacionadas (Martín, 
Cabero y De Paz, 2008:341), lo cual corrobora la existencia de este tipo de validez (ver tabla 2).

La fiabilidad o confiabilidad indica el grado en que distintos ítems son coherentes entre sí y pueden utilizarse para medir una misma magnitud (Jérez, 2001, citado por Calderón, 2008:73), es decir, es una medida de la homogeneidad; si los distintos ítems de un instrumento pretenden medir un mismo concepto, es de esperar que las respuestas estén relacionadas entre sí. En este caso, para comprobar el grado de confiabilidad del instrumento se calculó el coeficiente de Cronbach cuyo valor fue de 0,879 para todos los ítems, lo cual indica que hay consistencia interna en el instrumento.

Tabla 2. Matriz de correlaciones ${ }^{\mathrm{a}}$.

\begin{tabular}{|c|c|c|c|c|c|c|c|c|c|c|c|c|}
\hline & EXCON & SATIN & EFEAN & PERSE & CONF & CONT & DISP & REP & CAS & COSIR & EGOI & RET \\
\hline EXCON & 1 &, 320 &, 138 &, 383 &, 040 &, 359 &, $573^{*}$ &, 411 &, 255 &, 462 &, $589^{*}$ &, $561^{*}$ \\
\hline SATIN &, 320 & 1 &, 464 &, 259 &, 245 &, 437 &, $399^{*}$ &, 336 &, $528^{*}$ &, 296 &, 440 &, 348 \\
\hline EFEAN &, 138 &, 464 & 1 &, 473 &, $607^{*}$ &, $545^{*}$ &, $751^{* *}$ &, 153 &, 276 &, 454 &, 412 &, $666^{*}$ \\
\hline PERSE &, 383 &, 259 &, 473 & 1 &,- 064 &, $565^{*}$ &, 426 &, 340 &, 389 &, 016 &, 232 &, $523^{*}$ \\
\hline CONF &, 040 &, 245 &, $607^{*}$ &,- 064 & 1 &, 184 &, $501^{*}$ &,- 093 &, 101 &, 255 &, 300 &, 331 \\
\hline CONT &, 359 &, 437 &, $545^{*}$ &, $565^{*}$ &, 184 & 1 &, $614^{*}$ &, $540^{*}$ &, 471 &, 311 &, $594^{*}$ &, 470 \\
\hline DISP &, $573^{*}$ &, 399 &, $751^{*}$ &, 426 &, $501^{*}$ &, $614^{*}$ & 1 &, 406 &, 181 &, $704^{*}$ &, $580^{*}$ &, $709^{*}$ \\
\hline REP &, 411 &, 336 &, 153 &, 340 &,- 093 &, $540^{*}$ &, 406 & 1 &, $583^{*}$ &, 187 &, $500^{*}$ &, 094 \\
\hline CAS &, 255 &, $528^{*}$ &, 276 &, 389 &, 101 &, 471 &, 181 &, $583^{*}$ & 1 &,- 122 &, 260 &, 264 \\
\hline COSIR &, 462 &, 296 &, 454 &, 016 &, 255 &, 311 &, $704^{* *}$ &, 187 &,- 122 & 1 &, $605^{*}$ &, $548^{*}$ \\
\hline EGOI &, $589^{*}$ &, 440 &, 412 &, 232 &, 300 &, $594^{*}$ &, $580^{*}$ &, $500^{*}$ &, 260 &, $605^{*}$ & 1 &, $547^{*}$ \\
\hline RET &, $561^{*}$ &, 348 &, $666^{*}$ &, $523^{*}$ &, 331 &, 470 &, $709^{* *}$ &, 094 &, 264 &, $548^{*}$ &, $547^{*}$ & 1 \\
\hline
\end{tabular}

*. La correlación es significante al nivel 0,05 (bilateral).

**. La correlación es significativa al nivel 0,01 (bilateral).

a. Determinante $=3,544 \mathrm{E}-005$.

Fuente: elaboración propia a partir de las encuestas.

\subsection{Tratamiento estadístico}

El procesamiento, análisis e interpretación de la información se realizó con el programa estadístico SPSS y comprendió el estudio descriptivo de las variables estudiadas, las correlaciones entre las variables analizadas y las pruebas de rachas e independencia 
chi-cuadrado con el fin de caracterizar su comportamiento (Martín et al., 2008:29, 124); posteriormente se empleó la técnica de escalamiento multidimensional del análisis multivariante, que es una técnica de interdependencia que permite determinar la imagen relativa percibida de un conjunto de objetos sobre los que los individuos desarrollan percepciones (Valderrey, 2010:173), encontrar la estructura subyacente en un conjunto de medidas de proximidad/distancia entre objetos de cualquier tipo: países, marcas comerciales, relaciones de parentesco, empresas, descriptores de calidad, etc. (Guisande, Vaamonde y Barreiro, 2011:743), resumir la información para describirla más fácilmente reduciendo las dimensiones o variables (De la Garza, Morales y González, 2013:332) y encontrar una manera de condensar la información contenida en una serie de variables originales a una serie más pequeña de dimensiones compuestas o valores teóricos (factores) nuevos con una mínima pérdida de información (Hair, Anderson, Tathan y Black, 1999:280).

\section{Análisis de resultados}

\subsection{Análisis descriptivo}

El análisis descriptivo incluyó el estudio de las 12 variables (ver tabla 1) correspondientes a los prejuicios y errores en la toma de decisiones presentados por Robbins y Coulter (2010:133), cuando afirmaron que los gerentes al tomar decisiones no sólo utilizan su propio estilo, sino que es posible que empleen reglas empíricas o heurísticas para simplificar este proceso. La heurística puede resultar útil, ya que ayuda a darle sentido a la información compleja, incierta y ambigua (Mencl \& May, 2009; Langley, Mintzberg, Pitcher, Posada \& Macary, 1995; Simon, 1987; González, 2012; Miller \& Ireland, 2005, y Ramsés y Obuchi, 2010); sin embargo eso no significa que dichas reglas sean confiables ya que pueden llevar a errores en el procesamiento y evaluación de la información. Las siguientes son las frecuencias de las variables analizadas en las 16 organizaciones objeto de estudio.

En el gráfico 1 se observan las respuestas con respecto a la variable "error de exceso de confianza", donde se encuentra que el 31\% de las empresas estudiadas se mostró "de acuerdo y muy de acuerdo" con respecto a que en su proceso decisorio se incurría en el error de exceso de confianza. El 50\% de ellas se inclinó por la respuesta "en desacuerdo" y el 19\% se manifestó en los términos "ni de acuerdo, ni en desacuerdo". Como se infiere de estos datos, un considerable número de los ejecutivos de estas organizaciones (31\%), piensan que saben más sobre lo que hacen o mantienen visiones positivas no realistas de sí mismos y de su desempeño. 
Gráfico 1. Error de exceso de confianza.

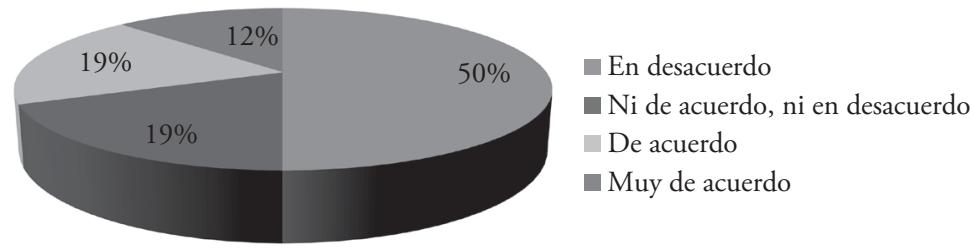

Fuente: los autores a partir de encuestas a empresas.

El gráfico 2, con relación al "error de satisfacción inmediata", refleja que el 75\% de los directivos de estas empresas se manifestó "de acuerdo y muy de acuerdo" en la obtención de beneficios inmediatos para evitar costos. Para estas personas, las opciones que brindan resultados rápidos resultan más atractivas que aquellas que generan resultados a futuro.

Gráfico 2. Error de satisfacción inmediata.

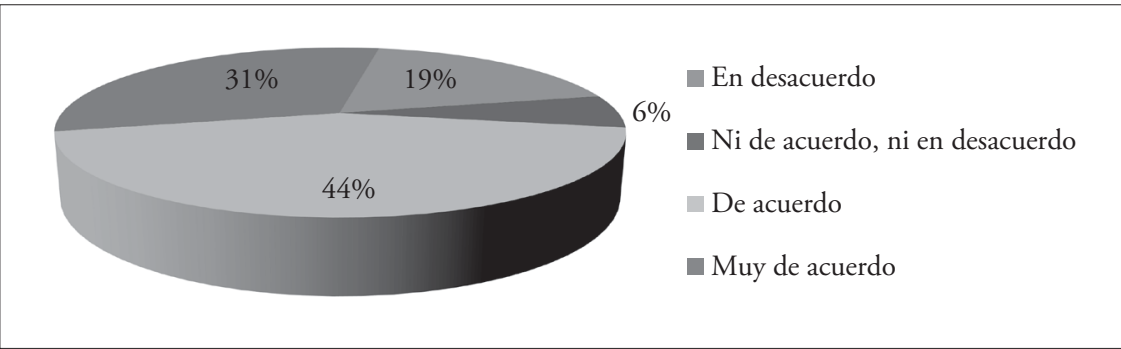

Fuente: los autores a partir de encuestas a empresas.

Sobre el "efecto ancla", el gráfico 3 muestra que el 50\% de estas organizaciones está "de acuerdo y muy de acuerdo" en utilizar esta variable como patrón en el proceso de toma de decisiones, es decir, los tomadores de decisiones se obsesionan con información inicial como punto de partida y luego, una vez fija, se equivocan en ajustar adecuadamente información posterior. Las primeras impresiones, ideas, precios y estimaciones conllevan ponderaciones injustificadas comparadas con la información recibida posteriormente. 
Gráfico 3. Efecto ancla.

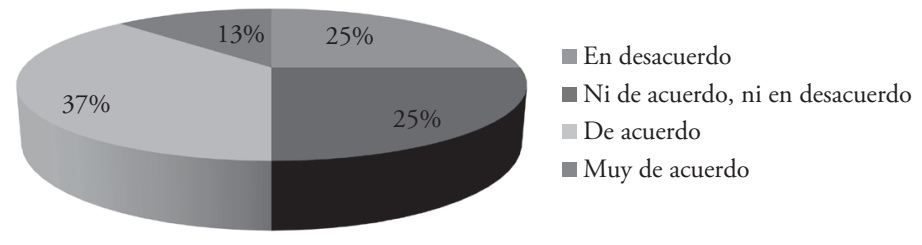

Fuente: los autores a partir de encuestas a empresas.

El gráfico 4 muestra que en el 57\% de estas empresas se mostró "de acuerdo y muy de acuerdo" con relación al criterio de la "percepción selectiva" en el momento de tomar decisiones. Los tomadores de decisiones organizan selectivamente e interpretan situaciones basadas en sus percepciones equivocadas.

Gráfico 4. Prejuicio de percepción selectiva.

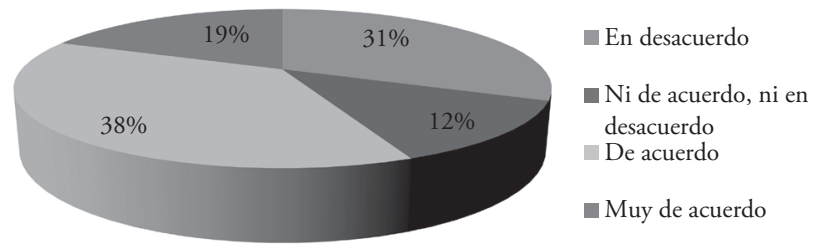

Fuente: los autores a partir de encuestas a empresas.

Con respecto al "prejuicio de confirmación", cuyos resultados se muestran en el gráfico 5 , en un porcentaje considerable de estas organizaciones $(75 \%)$ en la frecuencia "de acuerdo y muy de acuerdo", se viene utilizando este aspecto como fundamento en el diseńo de las decisiones. Los administradores tienden a aceptar literalmente la información que confirma sus ideas preconcebidas y son críticos y escépticos con la información que pone en duda estas ideas.

Gráfico 5. Prejuicio de confirmación.

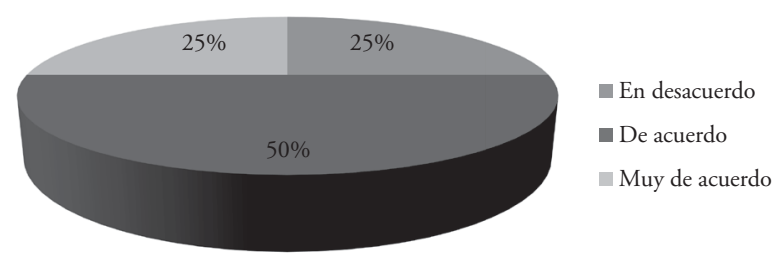

Fuente: los autores a partir de encuestas a empresas. 
Según el gráfico 6, con relación al "prejuicio de contextualización", los ejecutivos del $88 \%$ de estas empresas se mostraron "de acuerdo y muy de acuerdo" en resaltar ciertos aspectos de una situación y excluir otros. Al prestar atención a aspectos específicos del contexto y resaltarlos, y al mismo tiempo minimizar u omitir otros, distorsionan lo que ven y crean puntos de referencia incorrectos.

Gráfico 6. Prejuicio de contextualización ${ }^{8}$.

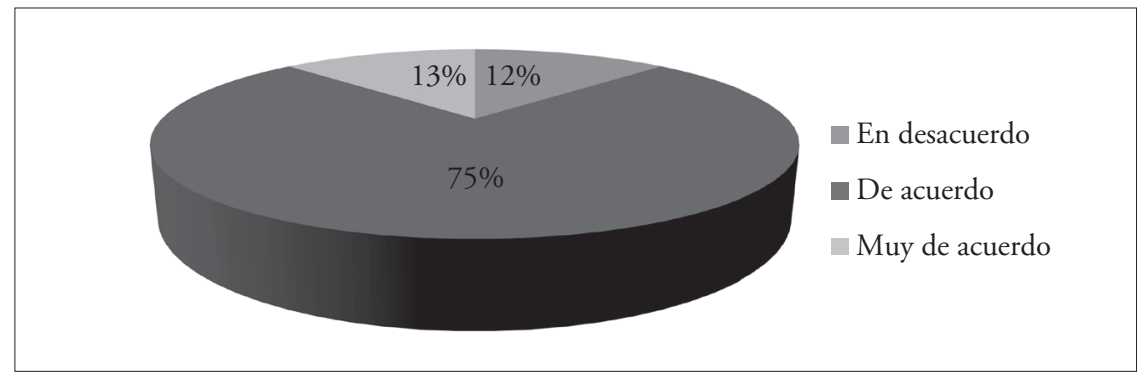

Fuente: los autores a partir de encuestas a empresas.

Según el gráfico 7, el 63\% de los gerentes de estas organizaciones en la frecuencia "de acuerdo y muy de acuerdo", incurren en el "prejuicio de disponibilidad", es decir, los tomadores de decisiones tienden a recordar los eventos más recientes y vívidos en su memoria. Este prejuicio distorsiona su capacidad de recordar eventos de manera objetiva y da como resultado juicios y estimaciones probabilísticas distorsionadas.

Gráfico 7. Prejuicio de disponibilidad.

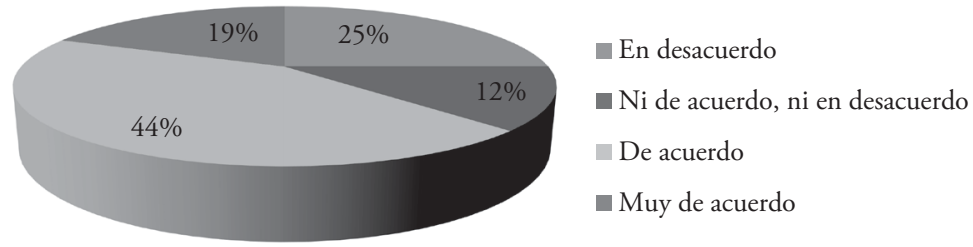

Fuente: los autores a partir de encuestas a empresas.

Sobre el "error de representación", en el gráfico 8 el 57\% de los administradores dijo estar "de acuerdo y muy de acuerdo" en utilizar esta variable en sus decisiones. Los gerentes que cometen este error encuentran analogías y ven situaciones idénticas donde no existen. 
Gráfico 8. Error de representación.

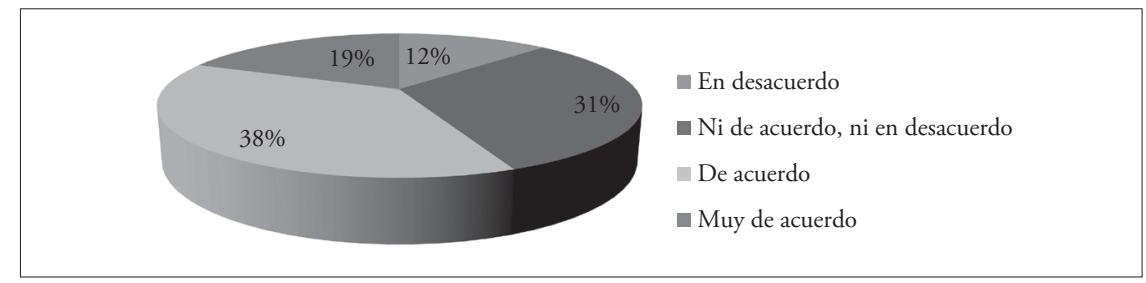

Fuente: los autores a partir de encuestas a empresas.

En el gráfico 9, se presentan los resultados correspondientes al "error de casualidad", donde se encuentra que el $56 \%$ de los tomadores de decisiones se manifiestan "de acuerdo y muy de acuerdo" con este aspecto. Este error ocurre cuando los ejecutivos intentan darle sentido a eventos casuales; lo hacen debido a que la mayoría tiene problemas para lidiar con el azar, aunque los eventos casuales le ocurren a toda la gente y no hay algo que pueda hacerse para predecirlos.

Gráfico 9. Error de casualidad.

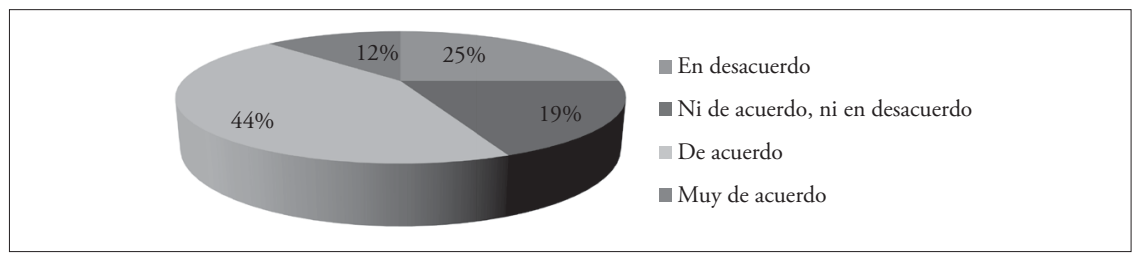

Fuente: los autores a partir de encuestas a empresas.

El 44\% de los empresarios, en la frecuencia de acuerdo y muy de acuerdo, según el gráfico 10, incurren en el "error de los costos irrecuperables", es decir, olvidan que las decisiones actuales no corrigen el pasado; de forma incorrecta se fijan en pérdidas anteriores de tiempo, dinero o esfuerzo en lugar de hacerlo en consecuencias a futuro. En vez de ignorar los costos irrecuperables, no los olvidan.

Gráfico 10. Error de los costos irrecuperables.

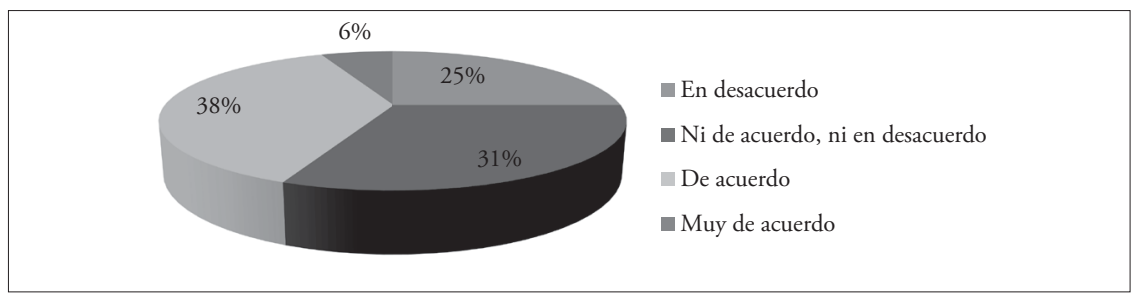

Fuente: los autores a partir de encuestas a empresas. 
El comportamiento "egoísta", cuyos resultados se reflejan en el gráfico 11, muestra que en las escalas de medición "de acuerdo y muy de acuerdo", se encuentran el 69\% de los directivos de las empresas estudiadas. Este hecho se pone de manifiesto cuando quienes toman decisiones se adjudican el crédito por sus triunfos y culpan a factores externos por los fracasos.

Gráfico 11. Egoísmo.

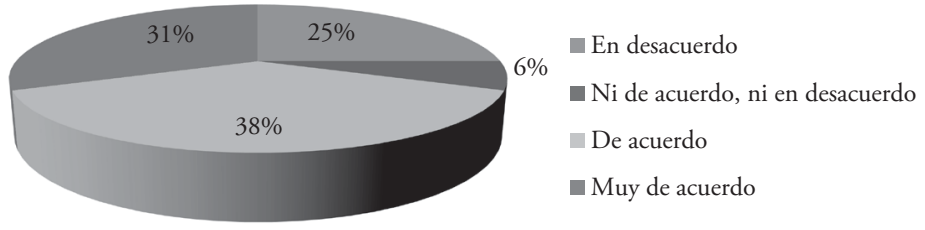

Fuente: los autores a partir de encuestas a empresas.

El gráfico 12 muestra que 50\% de los directivos de estas empresas se expresó estar "de acuerdo y muy de acuerdo" con la "predisposición a la retrospectiva" en sus decisiones, es decir, ellos tienden a creer falsamente, luego de que saben el resultado de un evento, que pudieron haberlo predicho acertadamente.

Gráfico 12. Predisposición a la retrospectiva.

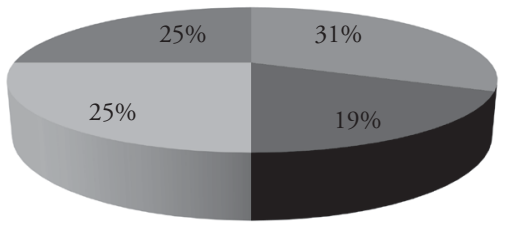

En desacuerdo

- Ni de acuerdo, ni en desacuerdo

De acuerdo

Muy de acuerdo

Fuente: los autores a partir de encuestas a empresas.

De los estadísticos descriptivos anteriores, se puede concluir que todos los errores y prejuicios en la toma de decisiones destacados por Robbins y Coulter (2010:133), presentan frecuencias altas en las escalas "de acuerdo y muy de acuerdo" y que significa que la administración en estas organizaciones tiene una alta inclinación por la utilización de técnicas intuitivas, "irracionales", subjetivas y en general heurísticas a la hora de tomar decisiones. Lo cual coincide con las investigaciones presentadas en el marco teórico en el sentido de que es prácticamente imposible desligar los métodos racionales, normativos o cuantitativos de los asociados con procesos sicológicos, conductivos o intuitivos. 


\subsection{Correlaciones}

En la tabla 2, se encuentra la matriz de correlaciones en la cual puede observarse que prácticamente todas las variables sometidas a prueba están correlacionadas positivamente según su determinante que es muy bajo $(3,544 \mathrm{E}-005)$, y que da cuenta de la intensidad de esas correlaciones (Martín, Cabero y De Paz, 2008:341) e incluso un buen número de ellas presentan correlaciones significativas al $5 \%$. Basados en estos resultados se procedió a contrastar la hipótesis $\mathbf{H}_{01}$ : "los prejuicios y errores en la toma de decisiones están correlacionados positivamente en las organizaciones objeto de estudio". Al respecto se encontró evidencia significativa para aceptar esta hipótesis y concluir que los 12 prejuicios y errores comunes en la toma de decisiones, que plantearon Robbins y Coulter (2010), están correlacionados positivamente en las organizaciones analizadas, lo cual puede generar que muchos de ellos, si no todos, se presenten en forma paralela en estas empresas.

Sobre el contraste de la hipótesis nula $\mathbf{H}_{02}$ : "cada uno de los errores y prejuicios tienen el mismo nivel de correlación en el proceso de toma de decisiones en estas organizaciones", se observó que muchos factores presentan niveles de asociación altos; entre ellos se destacan: exceso de confianza con disponibilidad, egoísmo y retrospectiva; satisfacción inmediata con casualidad; efecto ancla con confirmación, contextualización, disponibilidad y retrospectiva; percepción selectiva con contextualización y retrospectiva; confirmación con efecto ancla y disponibilidad; contextualización con efecto ancla, percepción selectiva, disponibilidad, representación y egoísmo; disponibilidad con exceso de confianza, efecto ancla, confirmación, contextualización, costos irrecuperables, egoísmo y retrospectiva; representación con contextualización, casualidad y egoísmo; casualidad con satisfacción inmediata y representación; costos irrecuperables con disponibilidad, egoísmo y retrospectiva; egoísmo con exceso de confianza, contextualización, disponibilidad, representación, costos irrecuperables y retrospectiva, y retrospectiva con exceso de confianza, efecto ancla, percepción selectiva, disponibilidad, costos irrecuperables y egoísmo.

Igualmente existen muchas de estas variables que tienen niveles de significación medios y bajos como puede evidenciarse en la tabla 2, por consiguiente se rechaza la hipótesis nula y se da paso a la hipótesis alternativa $\mathbf{H}_{2}$ para significar que cada uno de los errores y prejuicios tienen diferente nivel de correlación en el proceso de toma de decisiones en estas organizaciones.

\subsection{Pruebas de independencia}

Según la prueba de rachas que se muestra en la tabla 3, los errores y prejuicios en la toma de decisiones son aleatorios e independientes, pues sus $p$-valores asociados a 
los estadísticos de contraste son mayores que los niveles de significancia " $\alpha$ " al 1, 5 y 10\% (Ferrán, A., 2001:44; Valderrey, P., 2010: 87 y Martín, Q. et al., 2008:422). De lo anterior se puede concluir que así estos factores tengan cabida en el marco decisional de estas organizaciones, como se demostró anteriormente, no puede afirmarse que estos errores y prejuicios tengan influencia entre sí, es decir, uno o algunos de ellos no condicionan la existencia e influencia sobre otros. Estos hallazgos permiten aceptar la hipótesis nula $\mathbf{H}_{03}$ y en consecuencia afirmar que los procesos intuitivos, subjetivos e "irracionales", que dentro de este contexto se refieren a los errores y prejuicios en la toma de decisiones, son independientes en las empresas analizadas.

Tabla 3. Prueba de rachas.

\begin{tabular}{|c|l|l|l|l|l|l|l|l|l|l|l|l|}
\hline & EXCON & SATIN & EFEAN & PERSEL & CONFIR & CONTEX & DISPON & REPRES & CASUAL & COSIRR & EGOIS & RETROS \\
\hline $\begin{array}{c}\text { Valor de } \\
\text { prueba }^{2}\end{array}$ & 2,9375 & 3,8750 & 3,3750 & 3,4375 & 3,7500 & 3,8750 & 3,5625 & 3,6250 & 3,4375 & 3,2500 & 3,7500 & 3,4375 \\
\hline $\begin{array}{c}\text { Casos }<\text { Vr. } \\
\text { prueba }\end{array}$ & 8 & 4 & 8 & 7 & 4 & 2 & 6 & 7 & 7 & 9 & 5 & 8 \\
\hline $\begin{array}{c}\text { Casos }>=\mathrm{Vr} \\
\text { de prueba }\end{array}$ & 8 & 12 & 8 & 9 & 12 & 14 & 10 & 9 & 9 & 7 & 11 & 8 \\
\hline Casos & 16 & 16 & 16 & 16 & 16 & 16 & 16 & 16 & 16 & 16 & 16 & 16 \\
\hline No.rachas & 7 & 9 & 11 & 11 & 7 & 5 & 5 & 7 & 9 & 7 & 9 & 11 \\
\hline Z &,- 776 & 1,061 &, 776 &, 855 & 0,000 & 0,000 & $-1,664$ &,- 724 & 0,000 &,- 724 &, 381 &, 776 \\
\hline Sig. &, 438 &, 289 &, 438 &, 392 & 1,000 & 1,000 &, 096 &, 469 & 1,000 &, 469 &, 703 &, 438 \\
\hline
\end{tabular}

Fuente: los autores a partir de encuestas a empresas.

Igualmente en la prueba chi-cuadrado que se presenta en la tabla 4, se corroboran los resultados hallados en la prueba de rachas en el sentido que todas las variables, a excepción del error "efecto ancla" y el prejuicio de "disponibilidad", son independientes con un $p$-valor de contraste de 0,037 , que es menor al nivel de significancia $\alpha=0,05$ (Ferrán, A., 2001:44; Valderrey, P., 2010:87 y Martín, Q. et al., 2008:422).

Tabla 4. Prueba de chi-cuadrado: efecto ancla-disponibilidad.

\begin{tabular}{|l|c|c|c|}
\hline & Valor & gl & Sig. Asint. (bilateral) \\
\hline Chi-cuadrado de Pearson & 17,841 & 9 &, 037 \\
\hline Razón de verosimilitudes & 17,798 & 9 &, 038 \\
\hline Asociación lineal por lineal & 8,463 & 1 &, 004 \\
\hline N de casos válidos & 16 & & \\
\hline
\end{tabular}

Fuente: los autores a partir de encuestas a empresas. 


\subsection{Análisis multidimensional}

\subsubsection{Mapa perceptual}

En el gráfico 13 se muestra el mapa perceptual correspondiente a la disposición espacial de las 12 variables relacionadas con los prejuicios y errores en la toma de decisiones. Se observa en primera instancia que todos los factores se encuentran dispersos en el plano, lo cual permite concluir la disparidad en la evaluación que las empresas concedieron a estos atributos. Aquellos puntos que se encuentran cerca entre ellos son los que tienen mayor asociación y viceversa, lo cual se corrobora con los resultados hallados en la matriz de correlaciones (tabla 2), en las pruebas de rachas e independencia (tablas 3 y 4 ) y en el análisis descriptivo (gráficos 1-12), en el cual todas las variables presentan calificaciones superiores al 35\% en las frecuencias "de acuerdo y muy de acuerdo", que dan cuenta del énfasis que los directivos de estas organizaciones conceden a los prejuicios en su proceso de toma de decisiones y que se ve reflejado en la distancia de estas variables con relación al punto de inercia. El mapa perceptual tiene como propósito mostrar el comportamiento subyacente de un conjunto de observaciones, por ejemplo factores muy alejados del origen (outliers) pueden obedecer a altas o bajas calificaciones o distancias Euclídeas en el plano. Dentro de este contexto los atributos "satisfacción inmediata" (SATIN), "confirmación" (CONFIR) y "exceso de confianza” (EXCON), son los más alejados del punto de inercia. Los dos primeros se interpretan como los aspectos más críticos y requieren atención inmediata. EXCON recibió la mejor calificación entre las 12 variables analizadas por lo cual debe mantenerse su comportamiento y en lo posible mejorarse.

De la misma forma se encuentra que las demás variables presentan distancias relativamente iguales con relación al punto de inercia, lo cual indica que todas las organizaciones las calificaron de manera similar y requieren ser mejoradas en el mediano plazo. Cabe destacar el comportamiento del atributo "contextualización" (CONTEX), que posee la mayor calificación negativa y que se encuentra relativamente cerca del origen y requiere atención inmediata. Igualmente se distingue un grupo de factores con distancias muy similares que coinciden con sus distribuciones de frecuencia (EFEAN, DISPON, EGOIS, COSIRR Y RETROS) y que tuvieron la misma percepción entre los directivos de las empresas y que reclaman su mejoramiento en el mediano plazo.

De otra parte el mapa perceptual expone dos dimensiones, la primera de ellas concentra las variables "exceso de confianza" (EXCON) y "percepción selectiva" (PERSEL), las cuales a su vez poseen las mayores puntuaciones en la matriz de coordenadas de los estímulos (ver tabla 5). Esta dimensión podría denominarse "confianza" e invita 
a direccionar todos los esfuerzos de las empresas para evitar al máximo este tipo de conductas. La segunda dimensión comprende las variables "satisfacción inmediata" (SATIN) y "casualidad" (CASUAL), que también poseen las mayores puntuaciones en la matriz de coordenadas de los estímulos de la tabla 5. Esta dimensión podría recibir el nombre de "casualidad e inmediatez" y exige revisar estos comportamientos para colocarlos en contexto con la realidad organizacional.

Volviendo a la primera dimensión se distinguen allí los atributos más asociados con la gestión de la información situados a la izquierda del gráfico 13 (satisfacción inmediata "SATIN", contextualización "CONTEX", confirmación "CONFIR", efecto ancla "EFEAN", disponibilidad "DISPON", egoísmo "EGOIS" y costos irrecuperables "COSIRR"), de los factores relacionados con la incertidumbre ubicados a la derecha del mismo gráfico (casualidad "CASUAL", representación "REPRES”, percepción selectiva "PERSEL", retrospectiva "RETROS" y exceso de confianza "EXCON"). Se destacan aquí dos parámetros fundamentales a la hora de tomar decisiones: la disponibilidad de información confiable y la incertidumbre propia del ambiente organizacional, que requieren la implementación de técnicas racionales o métodos cuantitativos en forma paralela con los procedimientos heurísticos para el mejoramiento del proceso decisional en las organizaciones.

Por otra parte, la segunda dimensión distingue entre las variables asociadas con la confianza e información situadas abajo del gráfico 13 (confirmación "CONFIR", efecto ancla "EFEAN", disponibilidad "DISPON", egoísmo "EGOIS", costos irrecuperables "COSIRR", retrospectiva "RETROS" y exceso de confianza "EXCON"), de los atributos relacionados con el contexto y la casualidad ubicados en la parte de arriba del mismo gráfico (satisfacción inmediata "SATIN", contextualización "CONTEX", casualidad "CASUAL", representación "REPRES” y percepción selectiva "PERSEL"). Los aspectos comprendidos dentro de estas dimensiones llaman la atención de las directivas de estas organizaciones, para que fortalezcan la calidad de la información que utilizan frecuentemente en sus decisiones e incorporen técnicas prescriptivas o normativas que complementen los procesos intuitivos o heurísticos y así mejoren ostensiblemente su proceso de toma de decisiones. El stress para la muestra de estas empresas fue de 0.19064 (más cercano a cero que a uno) y que se considera aceptable, y el RSQ fue de 0.78724 que está cerca de 1 y que es bueno. Por lo tanto la solución del procedimiento ALSCAL mediante INDSCAL para el escalamiento multidimensional (MDS) en diferencias individuales se considera bueno (Guisande, Vaamonde y Barreiro et al., 2011:743-744; Valderrey, 2010:177 y Hair et al., 1999:564). 
Gráfico 13. Mapa perceptual errores en la toma de decisiones.

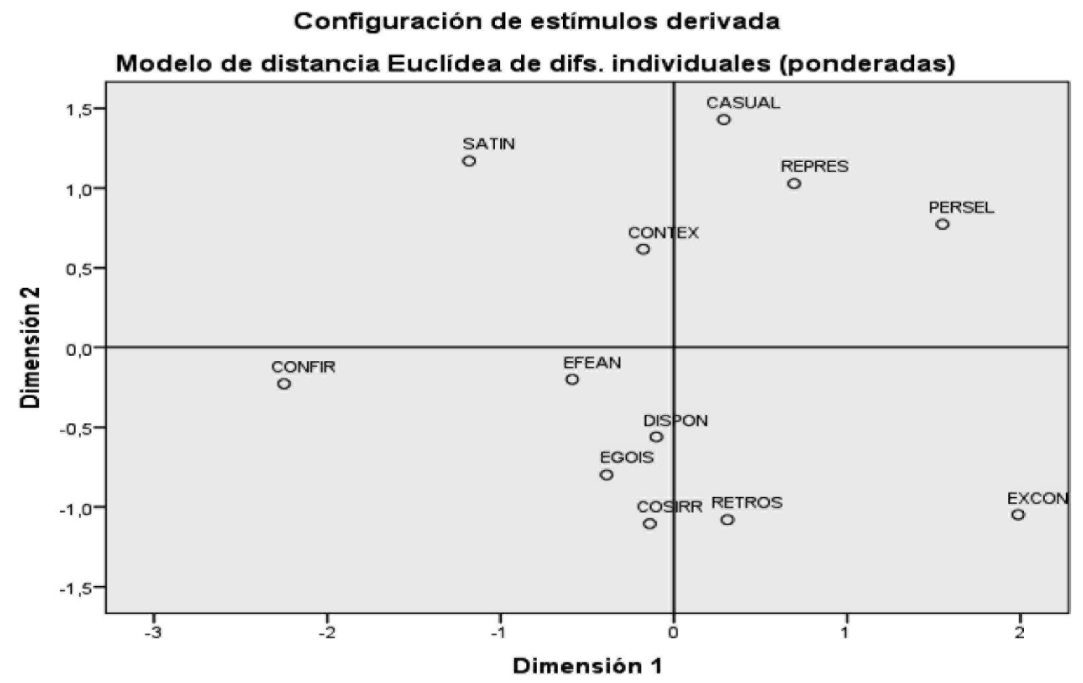

Tabla 5. Coordenadas de los estímulos.

\begin{tabular}{|c|c|c|c|}
\hline \multicolumn{5}{|c|}{ Stimulus Coordinates } \\
\hline Dimension & & & \\
\hline Stimulus & Stimulus & 1 & \\
\hline Number & Name & & $-1,0481$ \\
\hline 1 & EXCON & 1,9859 & 1,1702 \\
\hline 2 & SATIN & $-1,1816$ & $-0,1997$ \\
\hline 3 & EFEAN & $-0,5871$ & 0,7731 \\
\hline 4 & PERSEL & 1,5499 & $-0,2275$ \\
\hline 5 & CONFIR & $-2,2499$ & 0,6175 \\
\hline 6 & CONTEX & $-0,1770$ & $-0,5605$ \\
\hline 7 & DISPON & $-0,1018$ & 1,0278 \\
\hline 8 & REPRES & 0,6937 & 1,4305 \\
\hline 9 & CASUAL & 0,2872 & $-1,1051$ \\
\hline 10 & COSIRR & $-0,1395$ & $-0,7980$ \\
\hline 11 & EGOIS & $-0,3883$ & $-1,0802$ \\
\hline 12 & RETROS & 0,3086 & \\
\hline
\end{tabular}

Fuente: los autores a partir de encuestas a empresas. 


\section{Conclusiones e implicaciones}

La investigación permitió establecer en primera instancia que los principios y errores en la toma de decisiones (Robbins y Coulter, 2010), tienen una fuerte influencia en la toma de decisiones en estas empresas y que además están correlacionados positivamente e incluso un número apreciable de ellos con niveles de significancia al 0,05 y 0,01 , es decir, por lo menos desde el punto de vista de la asociatividad el perfil de estas empresas con relación a las variables estudiadas es muy similar. Igualmente la evidencia empírica demostró que el grado de correlación de los errores y prejuicios en la toma de decisiones en estas organizaciones es diferente, lo cual señala posturas heterogéneas de sus líderes con respecto a los atributos analizados y que se corrobora con el análisis descriptivo, al encontrarse que las respuestas a las doce variables en las frecuencias "de acuerdo y muy de acuerdo" oscilan entre el 44\% y el 88\%. Así mismo se encontró que los prejuicios y errores en la toma de decisiones son independientes entre sí, lo cual significa que no existen condicionamientos para la coexistencia de ellos en estas empresas.

Como conclusión general, los hallazgos permitieron establecer que estas empresas de manera tradicional, han dado gran importancia al empleo de los procesos intuitivos, subjetivos, e "irracionales" dentro de sus procesos de toma de decisiones sobre los modelos formales, lo cual podría explicarse como consecuencia del desconocimiento que sus administradores tienen acerca de las herramientas formales para este proceso o que su contexto decisional es muy estable o estructurado (Simon, 1965:31, 32). Este estado de cosas hace reflexionar acerca del desempeño de las empresas estudiadas con respecto al proceso de toma de decisiones, el cual se enfoca desde dos perspectivas, una racional que comprende la lógica y los modelos formales y una descriptiva asociada con procesos sicológicos, conductas e intuiciones.

Este hecho no se evidenció en estas organizaciones ya que han privilegiado lo intuitivo sobre lo formal, sin tener en cuenta que ambos enfoques se solapan y que los modelos formales del enfoque normativo necesitan aproximaciones intuitivas para ajustar ciertos parámetros y supuestos; $\mathrm{y}$ las decisiones de tipo descriptivo recurren a modelos formales y semiformales para entender y facilitar la toma de decisiones desde el punto de vista conductual. Al decidir se ponen en juego todas las facultades humanas: desde la racionalidad hasta la intuición y las emociones; desde la naturaleza gregaria del ser humano, que le permite utilizar su inteligencia colectiva y colaborativa, hasta el aislamiento del pensador solitario; desde el pensamiento crítico hasta la alegre improvisación de las tormentas de ideas; desde el anhelo de percepción hasta la propensión a cometer errores que permiten descubrir soluciones novedosas e insospechadas (Ramsés y Obuchi, 2010:23, y Miller y Ireland, 2005:19, 28). 
Este estudio abre las posibilidades para profundizar en un tema de tanta importancia para las organizaciones, máxime cuando de manera permanente se están tomando decisiones desde las más sencillas hasta las más complejas que incluso pueden comprometer la supervivencia de una empresa. Trabajos posteriores podrían considerar muestras más grandes en el mismo sector de los servicios para observar la consistencia de los resultados y extender el estudio a diferentes sectores, incluyendo por ejemplo al sector manufacturero.

\section{Bibliografía}

Calderón, Gregorio; Álvarez, Claudia Milena y Naranjo, Julia Clemencia (2008). Estrategia empresarial y gestión humana en empresas colombianas. Bogotá, Unibiblos, 152 pág.

Castillo, Mario (2006). Toma de decisiones en las empresas: entre el arte y la técnica. Bogotá, Ediciones Uniandes, 466 pág.

Círigo, Alberto (2010). El poder de la intuición. Disponible en:

http://web.b.ebscohost.com/ehost/pdfviewer/pdfviewer? $v i d=$ 8\&sid=8f9c4976-2410-4c2e-b7fd-9db60c9eebd2\%40sessionmgr114\&h $\mathrm{id}=118$.

Dane, Erik \& Pratt, Michael (2007). Exploring intuition and its role in managerial decision making. Disponible en: http://www.jstor.org/stable/20159279.

De La Garza, Jorge; Morales, Blanca Nieves y González, Beatriz Adriana (2013). Análisis estadístico multivariante. Un enfoque teórico y práctico. McGraw Hill, México, 712 pág.

Deslauriers, Jean-Pierre (2004). Investigación cualitativa guía práctica. Colombia, Papiro, Pereira, 142 pág.

Ferrán, Magdalena (2001). SPSS para Windows, análisis estadístico. Madrid, McGrawHill, 421 pág.

Forest, Joelle \& Mehier, Caroline (2001). John R. Commons and Herbert A. Simon on the concept of rationality. Disponible en: http://www.jstor.org/stable/4227692.

Franklin, Enrique (2011). Toma de decisiones empresariales. Disponible en: http:// web.b.ebscohost.com/ehost/pdfviewer/pdfviewer?vid=8\&sid=8f9c49762410-4c2e-b7fd-9db60c9eebd2\%40sessionmgr1 14\&hid=118.

González, Milko (2012). La intuición y el pensamiento gerencial. Disponible en: http:// web.b.ebscohost.com/ehost/pdfviewer/pdfviewer?vid=8\&sid=8f9c49762410-4c2e-b7fd-9db60c9eebd2\%40sessionmgr1 14\&hid=118. 
Guisande, Castor; Vaamonde, Antonio y Barreiro, Aldo (2011). Tratamiento de datos con $R$, statistica y SPSS. Madrid, España, Díaz de Santos, 978 pág.

Hair, Joseph F.; Anderson, Rolph E.; Tatham, Ronald L. y Black, William C. (1999). Análisis multivariante. 5a ed., Madrid, Prentice Hall, 799 pág.

Hernández, Roberto; Fernández, Carlos y Baptista, María Del Pilar (2010). Metodología de la investigación, México, McGraw Hill, 613 pág.

Langley, Ann; Mintzberg, Henry; Pitcher, Patricia; Posada, Elizabeth \& Macary, Jan Saint (1995). Opening up decision making: the view from the black stool. Disponible en http://www.jstor.org/stable/2635251.

Machado, Álvaro; Van, Adrián \& Britto, Luiz (2013). A new framework to measure intuitiveness in decisions problems. Disponible en:

http://web.b.ebscohost.com/ehost/pdfviewer/pdfviewer?vid=8\&sid=8f9c4976-2410 4c2e-b7fd-9db60c9eebd2\%40sessionmgr114\&hid=118.

March, James y Simon, Herbert (1981). Teoría de la organización. 5a ed., Barcelona, Ediciones Ariel, 285 pág.

Martín, Quintín; Cabero, María Teresa T. y De Paz, Yanira Del Rosario (2008). Tratamiento estadístico de datos con SPSS prácticas resueltas y comentadas, Madrid, Thomson, 596 pág.

Mencl, Jennifer \& May, Douglas (2009). The effects of proximity and empathy on ethical decision-making: an exploratory investigation. Disponible en: http://www.jstor.org/stable/41315826.

Miller, C. Chet \& Ireland, R. Duane (2005). Intuition in strategic decision making: friend or foe in the fast-paced 21st century? Disponible en: http://www.jstor.org/stable/4166150.

Mintzberg, Henry; Brian, James y Voyer, John (1997). El proceso estratégico: conceptos, contextos y casos, México, Prentice Hall, 641 pág.

Murphy, John W. (1992). Reason, bounded rationality and the lebenswelt: socially sensitive decision making. Disponible en: http://www.jstor.org/stable/3487312.

Ramsés, Milko y Obuchi, Richard K. (2010). La ciencia, el arte y la psicología de decidir. Disponible en: http://web.b.ebscohost.com/ehost/pdfviewer/ pdfviewer? vid=8\&sid=8f9c4976-2410-4c2e-b7fd-9db60c9eebd2\%40sess ionmgr114\&hid $=118$.

Regent, Pablo (2012). Dirigir: ¿intuición o método? Algunas reflexiones de verano. Disponible en:

http://web.b.ebscohost.com/ehost/pdfviewer/pdfviewer? $v$ vid=8\&sid=8f9c49762410-4c2e-b7fd-9db60c9eebd2\%40sessionmgr114\&hid=118. 
Rivera, Gerardo; Braunstein, Daniel \& Hall, Phillip (1981). Managerial information processing: a research review. Disponible en: http://www.jstor.org/ stable/2392604.

Robbins, Stephen y Coulter, Mary (2010). Administración. 10a ed., México, Pearson, 564 pág.

Simon, Henry (1965). Administrative decision making. Disponible en: http://www. jstor.org/stable/974005.

Simon, Henry (1987). Making management decisions: The role of intuition and emotion. Disponible en: http://www.jstor.org/stable/4164720.

Simon, Henry (1991). Bounded rationality and organizational learning. Disponible en: http://www.jstor.org/stable/2634943.

Tsang, Eric. W. K. (2004). Superstition and decision-making: contradiction or complement? Disponible en: http://www.jstor.org/stable/4166126.

Valderrey, Pablo (2010). SPSS 17. Extracción del conocimiento a partir del análisis de datos, México, Alfaomega-Ra-Ma, 463 pág.

\section{Cómo citar este artículo:}

Rubio, G. y Fierro, F. (2015). "La heurística y la toma de decisiones en empresas de servicios", Oikos No 38, 83-108, Escuela de Administración y Economía, Universidad Católica Silva Henríquez (UCSH), Santiago de Chile [http://ediciones.ucsh.cl/revistas.php]

Fecha de recepción: 16 / $01 / 2015$

Fecha de aceptación: 05 / 03 / 2015 